\title{
Promoted and Controllable Self-Assembly of Hydrolyzed Siloxane and Triblock Copolymer under Organic Polyhydroxy Acids
}

\author{
Jin Jun Li, ${ }^{\dagger}$ Zhen Mu, ${ }^{\dagger}$ Chi He, ${ }^{\dagger}$ Qin Hu, ${ }^{\dagger}$ Lan Dong Li, ${ }^{\dagger}$ Jie Cheng, ${ }^{\dagger}$ Zheng Ping Hao, ${ }^{*},{ }^{\dagger}$ and \\ Shi Zhang Qiao*," \\ Research Center for Eco-Environmental Sciences, Chinese Academy of Sciences, Beijing 100085, People's \\ Republic of China, and ARC Centre for Functional Nanomaterials, School of Engineering and Australian \\ Institute for Bioengineering and Nanotechnology, The University of Queensland, Brisbane QLD 4072, Australia
}

\begin{abstract}
Large-molecule polyhydroxy organic acids such as tartaric acid and citric acid were found to significantly promote the cooperative formation of mesoporous silica SBA-15 with homogeneous well-defined hexagonal mesostructure under much weaker acidities without addition of volatile inorganic acids; therefore a novel environmental benign route for SBA-15 synthesis was developed. It is suggested that the polar groups of polyhydroxy acid molecules can greatly strengthen the assembly interactions between the silica species and the triblock copolymer by providing hydrogen bonding. More interestingly, by controlling the strength of hydrogen-bonding interaction that is dependent on the concentration of carboxylic acids, the particle size of the product can be tailored. The silica with small particle size synthesized with a small amount of polyhydroxy acids was favorable support of palladium catalyst for the complete oxidation of volatile organic compounds in atmosphere, probably due to the facilitated diffusion of reactant molecules.
\end{abstract}

\section{Introduction}

By using pluronic triblock copolymer as template agent, mesoporous silica SBA-15 with large pore diameter, thick pore wall, and good thermal and hydrothermal stability was first synthesized by Zhao et al., ${ }^{1,2}$ which has aroused particular interest in the synthesis of mesoporous materials because of their potential versatile applications in the fields of catalysis, adsorption separation, microelectronics, chromatography, sensors, and so on. ${ }^{3-8}$ SBA-15 was generally synthesized in the presence of hydrochloric acid with a high a concentration of more than $1.6 \mathrm{M} .{ }^{1,2,9}$ The concentrated inorganic acids appeared to facilitate the rapid synthesis of periodic mesoporous materials by accelerating the hydrolysis of siloxane, promoting the condensation of silica species, as well as improving the hexagonal packing of the silica-surfactant hybrid micelles. ${ }^{9-13}$

However, the synthesis of SBA-15 under strong acidities has some shortcomings. For example, the corrosive solutions containing a high concentration of volatile inorganic acids are disadvantageous for large-scale production. Furthermore, strong acidities suppress the direct incorporation of heterogeneous atoms into the framework of the silica to obtain functionalized catalysts. ${ }^{14-16}$ Therefore, some attempts have been made to synthesize SBA-15 under moderate conditions. ${ }^{17-22}$ However, weak acidities seem difficult to afford quick phase separation of mesostructured composites,, 12 and some inorganic salts are often needed to promote the formation of ordered mesostructure. ${ }^{13,17,22-27}$ For example, Chen et al. ${ }^{17}$ synthesized mesoporous silica materials under acid-free conditions with the aid of salt and alcohol, and it was revealed that the formation of mesostructures depended on the concentrations of the additives and the copolymer. Nonetheless, it was noticed that the obtained mesoporous silica were still less ordered in comparison to the conventional SBA-15 synthesized under strong acidities, as most samples featured only one or two weak Bragg peaks in the X-ray diffraction (XRD) patterns. Some other

* To whom correspondence should be addressed. E-mail: zpinghao@ rcees.ac.cn (Z.P.H.); s.qiao@uq.edu.au (S.Z.Q.).

Chinese Academy of Sciences.

$¥$ The University of Queensland. researchers also demonstrated that the use of inorganic salts under acidic conditions could dramatically widen the domains of synthesis conditions to produce ordered structure. ${ }^{22-24}$ But there would be some potential problems in disposal of the wastewater containing a high concentration of inorganic salts after the synthesis. Some other efforts have been made to accelerate the hydrolysis of siloxane and the condensation of the resultant silica precursors. ${ }^{18-20}$ TMOS was sometimes used as a silica source instead of the extensively used TEOS because the former hydrolyzed more quickly. ${ }^{18,20}$ Fluoride ions were also often used to accelerate the hydrolysis of siloxane and promote the condensation of silica species. ${ }^{1,20,24}$ Fluoride could induce ordered hexagonal mesostructures in a wide acidity range of $\mathrm{pH} 0-9$ with tetramethyl orthosilicate (TMOS) as silica source ${ }^{20}$ and also enhance mesostructural ordering at values of up to $\mathrm{pH}$ 2.7 with Tetraethyl orthosilicate (TEOS) as silica source. ${ }^{19}$ But fluoride ions are one of the major concerns for environmental pollutants. Alternatively, sometimes prehydrolysis of TEOS under lower $\mathrm{pH}$ values was adopted to form silica oligomers or monomers before they were mixed with the template solution to start the assembly reactions. ${ }^{21,28-30}$

Recently, combined use of hydrochloric acid and other inorganic acids were employed for synthesis of SBA-15 under relatively moderate acidities. Luo et al. ${ }^{31}$ found that the addition of a small amount of heteropoly acids could afford a rapid synthesis of SBA-15 with a low concentration of hydrochloric acid, and the authors suggested that the heteropoly acids strengthened the assembly forces. In another work, it was found that the amount of hydrochloric acids could be greatly reduced when phosphoric acid was also present in the synthesis solution, ${ }^{32}$ and the obtained SBA-15 has significantly enlarged surface areas and pore volumes. It seemed that the phosphoric acid modified the ionic strength and $\mathrm{pH}$ of the environment around the template phase. ${ }^{32}$

An interesting work was done recently by Hsu et al., ${ }^{9}$ in which they used carboxylate-terminated triblock copolymer P123 as template agent to synthesize SBA-15 with additional intraparticle porosity. During the synthesis the terminal hydroxyl group of the Pluronic P123 needs to be first oxidized to the 
carboxyl group by potassium dichromate or potassium permanganate, and the synthesis could be carried out with a low hydrochloric acid concentration of $0.3 \mathrm{M}$. It was revealed that the carboxyl group could catalyze the hydrolysis of siloxane. ${ }^{9}$ In another work about synthesis of SBA- $15,{ }^{33}$ carboxylic acids were used to prehydrolyze TEOS, but the assembly still occurred in hydrochloric acid solution with high concentration of $2 \mathrm{M}$.

Herein, we will show the synthesis of SBA-15 using weak organic acids as a single component of the acid source. A single component of weak organic acids has rarely been used to adjust the acidities in the synthesis of SBA-15 because it is difficult for them to achieve strongly acidic conditions in the absence of additional inorganic acids. Nonetheless, we found that under moderate acidities at $\mathrm{pH}>1$ some large-molecule carboxylic acids with polyhydroxy can promote the formation of highquality mesoporous silica that is comparable to the conventional SBA-15 synthesized under strong acidities, although it was previously suggested that no ordered mesostructure could be formed under such acidities with the use of strong mineral acids. ${ }^{1,2}$ In this present work, various organic carboxylic acids were used as acid source, TEOS was used as silica source without prehydrolysis, and the addition of fluoride or other salts was also avoided. Interestingly, it was found that the size of the primary particle in the product could be controlled by varying the concentration of polyhydroxy carboxylic acids, and therefore the lengths of mesochannels could be tailored for special catalysis applications. ${ }^{34-36}$ Furthermore, the polyhydroxy carboxylic acids are in solid state and nonvolatile; thus, they can be more safely handled during the synthesis in comparison to the generally used hydrochloric acid. The synthesis processes are environmentally friendly with use of biodegradable carboxylic acids instead of fluoride or other salts as promoter. In addition, some products with controlled particle size showed an advantage in catalytic elimination of volatile organic compounds, probably due to facile mass transfer.

\section{Experimental Section}

2.1. Chemicals. The nonionic Pluronic triblock copolymer $\mathrm{P} 123\left(\mathrm{EO}_{20} \mathrm{PO}_{70} \mathrm{EO}_{20}, M_{\mathrm{n}}=5800\right)$ was purchased from SigmaAldrich. Tetraethyl orthosilicate (TEOS, containing $28 \%$ of $\mathrm{SiO}_{2}$ ) was purchased from Tianjin Fuchen Chemical Reagent Factory. Citric acid (99.9\%), acetic acid (99.9\%), oxalic acid (99.5\%), and palladium chloride (containing $58 \%$ of palladium) were purchased from Beijing Chemical Reagents Co. Tartaric acid $(99.5 \%)$ was purchased from Sinopharm Chemical Reagent Co., Ltd. All of the reagents were used as received without any further purification.

2.2. Material Preparation. The typical synthesis of mesoporous silica was similar to the general synthesis procedure of SBA-15 under strong acidities except for the moderately acidic conditions. $^{1,2} \mathrm{~A} 4 \mathrm{~g}$ amount of P123 was dissolved in $150 \mathrm{~g}$ of water, followed by adjusting the $\mathrm{pH}$ value of the solution in the range from 1 to 7 through adding a certain type of organic acid; then $8.5 \mathrm{~g}$ of TEOS was added to the acidic P123 solution under stirring. After continuous stirring for a half-hour, the mixture underwent the first aging at a low temperature of 35 ${ }^{\circ} \mathrm{C}$ for 1 day and the second aging in an autoclave at a high temperature of $100{ }^{\circ} \mathrm{C}$ for a subsequent 2 days. The resultant solid was filtered, dried at $100{ }^{\circ} \mathrm{C}$, and then calcined in air at $550{ }^{\circ} \mathrm{C}$ for $3 \mathrm{~h}$, thus forming the silica powder product. The acids used in this work included acetic acid, oxalic acid, tartaric acid, and citric acid. Correspondingly, the products were denoted as $\mathrm{MS}_{\mathrm{a}}-y, \mathrm{MS}_{\mathrm{ox}}-y, \mathrm{MS}_{\mathrm{t}}-y$, and $\mathrm{MS}_{\mathrm{c}^{-}}-y$, respectively, where $y$ denotes the $\mathrm{pH}$ value of the synthesis solution.
Mesoporous silica supported palladium catalysts with a loading of $0.3 \mathrm{wt} \%$ were prepared by incipient aqueous wetness impregnation method using palladium chloride as precursors of the catalytic phase. The impregnated solids were dried at $100{ }^{\circ} \mathrm{C}$ overnight, calcined in air at $500{ }^{\circ} \mathrm{C}$ for $3 \mathrm{~h}$, and then reduced in hydrogen flow at $480{ }^{\circ} \mathrm{C}$ for $1 \mathrm{~h}$ before activity evaluation.

2.3. Material Characterizations. Small-angle XRD patterns were recorded on a Siemens D5005 diffractometer at $40 \mathrm{KV}$ and $30 \mathrm{~mA}$ using $\mathrm{Cu} \mathrm{K \alpha}$ radiation. Transmission electron microscopy (TEM) images were taken on a Hitachi H-7500 electron microscope operating at an acceleration voltage of 80 $\mathrm{kV}$. Prior to observation, all samples were crushed, dispersed in ethanol, sonicated for $10 \mathrm{~min}$, and deposited on a microgrid to evaporate the solvent. Nitrogen adsorption/desorption isotherms at liquid nitrogen temperature were obtained on NOVA1200 gas sorption analyzer. The samples were degassed at $300{ }^{\circ} \mathrm{C}$ in a vacuum for more than $5 \mathrm{~h}$ before measurements. The Brumauer-Emmet-Teller (BET) surface areas were calculated on the basis of the linear part of the BET plot $\left(P / P_{0}\right.$ $=0.05-0.25)$. The total pore volumes were estimated according to nitrogen uptake at a relative pressure $\left(P / P_{0}\right)$ of ca. 0.99 . The pore size distributions (PSD) were derived from the desorption branch of the $\mathrm{N}_{2}$ isotherms using the Barrett-Joyner-Halenda (BJH) method. Infrared spectra were recorded on a Bruker Tensor 27 using diffuse reflectance infrared Fourier transform spectroscopy (DRIFT) techniques.

2.4. Catalytic Activity Evaluation. Catalytic activities toward the complete oxidation of benzene were evaluated in a continuous-flow fixed-bed reactor. An online gas chromatograph equipped with flame ionization detector (FID) was used to analyze the concentration of benzene in the feed and effluent gas. In each test run, $50 \mathrm{mg}$ of catalyst (40-60 mesh) was used, and the total flow rate was kept at $320 \mathrm{~mL} / \mathrm{min}$ with a benzene concentration of $1050 \mathrm{ppm}$.

\section{Results and Discussion}

3.1. Effect of Acidity and Acid Source on Mesostructure. To obtain high-quality SBA-15 with the use of strong inorganic acids, previous work has revealed that it is important that the synthesis is carried out at $\mathrm{pH}<1$; otherwise, no precipitation or formation of silica gel occurs. ${ }^{1,2}$ Organic acids are rarely used because they are hardly capable of achieving strongly acidic conditions. Unexpectedly, it was found in this work that some organic polyhydroxy acids such as tartaric acid and citric acid can actually induce very good mesostructural ordering under moderately acidic conditions. The XRD patterns of $\mathrm{MS}_{\mathrm{t}}$ silica synthesized at various $\mathrm{pH}$ values adjusted by tartaric acid are shown in Figure 1a. At $\mathrm{pH} \leq 2.8$, the $\mathrm{MS}_{\mathrm{t}}$ samples possess more than three Bragg peaks in their XRD profiles, which can be indexed as (100), (110), and (200) diffractions and reflect a two-dimensional hexagonal mesostructure with a space group of $P 6 \mathrm{~mm} .{ }^{1,2}$ Especially, for the samples synthesized at $\mathrm{pH} 1.4-1.8$, the additional (210) diffraction peak can be clearly resolved, indicating these materials have a high quality of mesostructural ordering (the long-range regularity of wall mesostructure) that is comparable to those synthesized under strongly acidic conditions induced by strong mineral acids. ${ }^{1,2}$ Figure $1 \mathrm{~b}$ shows the XRD patterns of $\mathrm{MS}_{\mathrm{ci}}$ silicas synthesized at various $\mathrm{pH}$ values adjusted by citric acid. Citric acid has similar effects on the mesostructural ordering of the product as tartaric acid does, namely, the obtained $\mathrm{MS}_{\mathrm{ci}}$ silicas synthesized at $\mathrm{pH} \leq 2.8$ possess fine mesostructural ordering with more than three diffraction peaks 

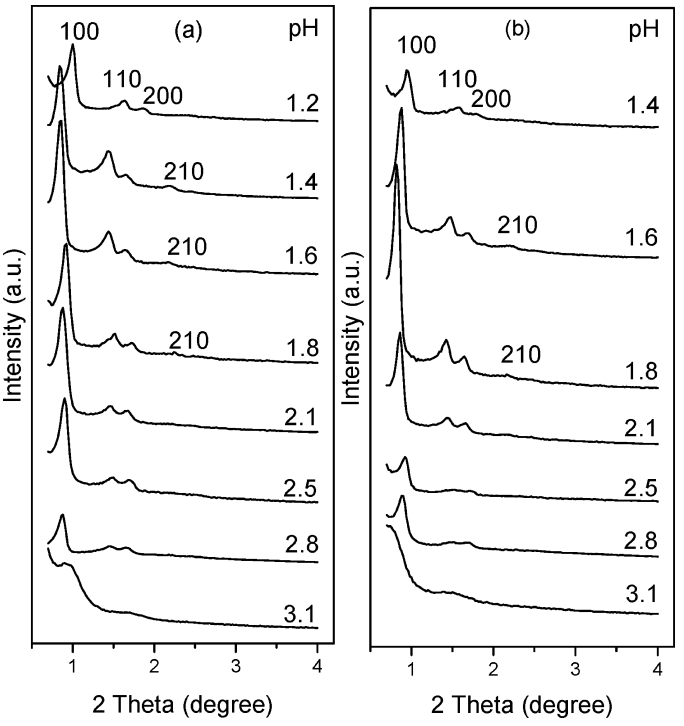

Figure 1. XRD patterns of silicas synthesized at various $\mathrm{pH}$ values adjusted by large-molecule polyhydroxy carboxylic acids: (a) $\mathrm{MS}_{\mathrm{t}}$ and (b) $\mathrm{MS}_{\mathrm{ci}}$.
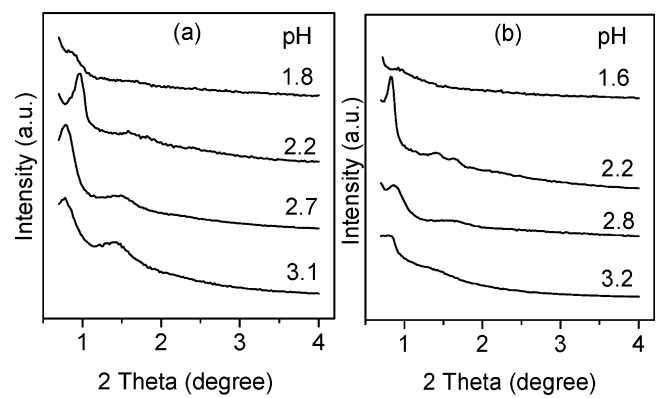

Figure 2. XRD patterns of silicas synthesized at various $\mathrm{pH}$ values adjusted by small-molecule carboxylic acids: (a) $\mathrm{MS}_{\mathrm{a}}$ and (b) $\mathrm{MS}_{\mathrm{ox}}$.

in the XRD patterns, and the additional (210) diffraction peak appears at $\mathrm{pH} 1.6-1.8$.

However, small-molecule organic acids, including acetic acid and oxalic acid, seemed to induce less ordering to the product. Figure 2 shows the XRD patterns of $\mathrm{MS}_{\mathrm{a}}$ and $\mathrm{MS}_{\mathrm{ox}}$ silicas that were synthesized at various $\mathrm{pH}$ values adjusted by acetic acid and oxalic acid, respectively. Although somewhat ordered structure can be formed within a narrow acidity range at $\mathrm{pH}>$ 2 , the diffraction intensity is much weaker than the cases when tartaric acid or citric acid were used. It seems that the optimal $\mathrm{pH}$ value is ca. 2.2, because three diffraction peaks can be resolved in both $\mathrm{MS}_{\mathrm{a}}-2.2$ and $\mathrm{MS}_{\mathrm{ox}}-2.2$, whereas, not more than two peaks can be found in other samples.

Figure 3 shows some TEM images of $\mathrm{MS}_{\mathrm{t}}$ and $\mathrm{MS}_{\mathrm{ci}}$ silicas synthesized at different $\mathrm{pH}$ values adjusted by using largemolecule polyhydroxy acids, which further corroborates the fine periodicity of mesopores in these samples. It can be seen from high-magnification images (Figure 3a,c,e,g) that the number of the packed channels in each silica particle synthesized at $\mathrm{pH}<$ 2 is substantially larger than that synthesized at $\mathrm{pH} 2.8$ for both $\mathrm{MS}_{\mathrm{t}}$ and $\mathrm{MS}_{\mathrm{ci}}$. It reveals that relatively higher concentration of organic acid favors the formation of an enlarged ordered domain. In addition, the low-magnification images (Figure $3 b, d, f, h$ ) show that the primary particles are fine rods or curved rods for all samples synthesized at $\mathrm{pH} \leq 2.8$, and the sizes of the rods (diameter and length) increase with decreasing $\mathrm{pH}$ values. For example, the rods of sample $\mathrm{MS}_{\mathrm{t}}-2.8$ are, on average, ca. 260 $\mathrm{nm}$ in diameter and ca. $1.3 \mu \mathrm{m}$ in length, whereas the diameter
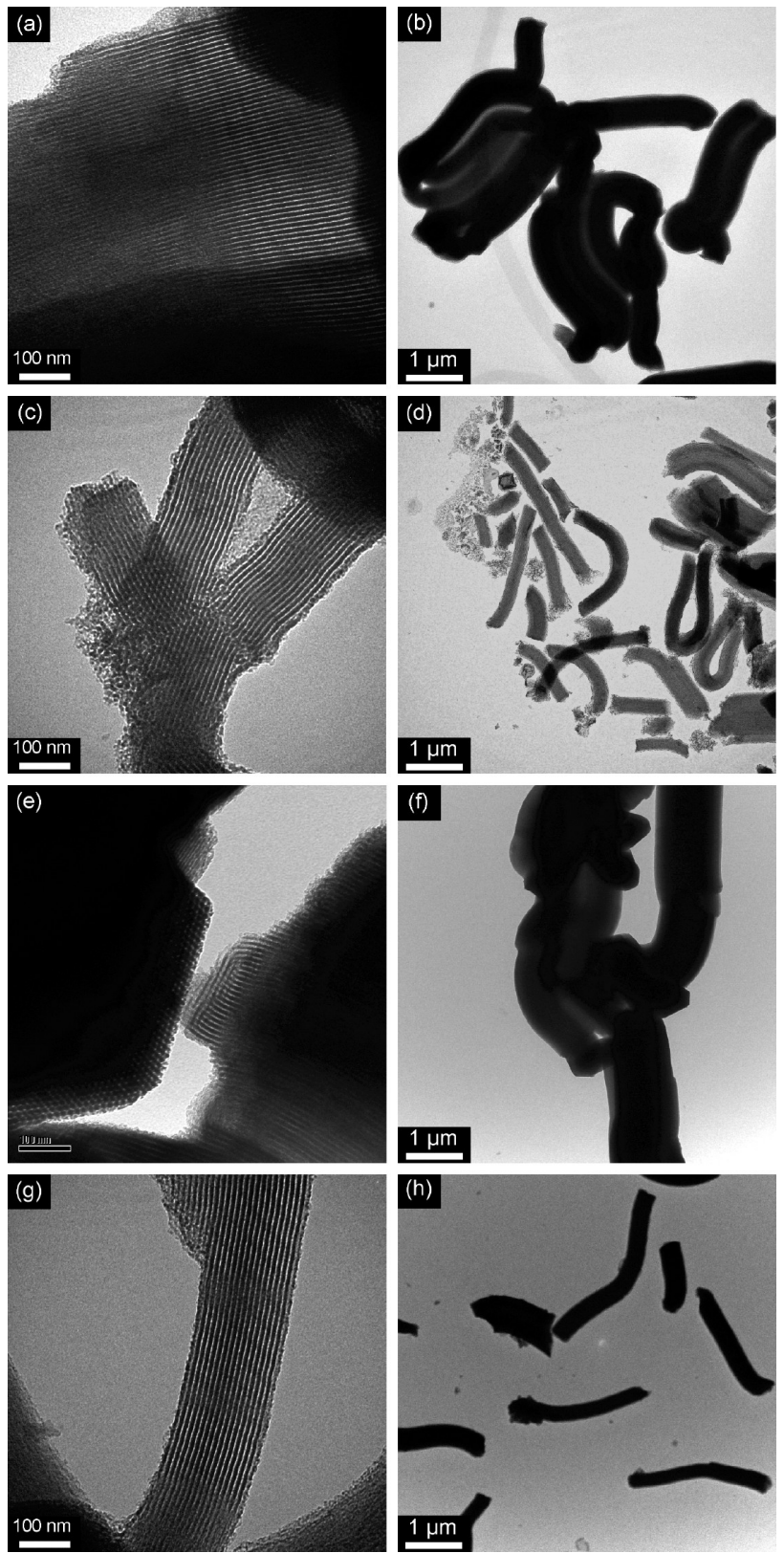

Figure 3. High-magnification TEM images of (a) $\mathrm{MS}_{\mathrm{a}}-1.6$, (c) $\mathrm{MS}_{\mathrm{t}}-2.8$, (e) $\mathrm{MS}_{\mathrm{ci}}-1.8$, and $(\mathrm{g}) \mathrm{MS}_{\mathrm{ci}}-2.8$ and low-magnification TEM images of (b) $\mathrm{MS}_{\mathrm{t}}-1.6$, (d) $\mathrm{MS}_{\mathrm{t}}-2.8$, (f) $\mathrm{MS}_{\mathrm{ci}}-1.8$, and (h) $\mathrm{MS}_{\mathrm{ci}}-2.8$.

and length of the rods for sample $\mathrm{MS}_{\mathrm{t}}-1.6$ increase to ca. 650 $\mathrm{nm}$ and ca. $3.4 \mu \mathrm{m}$, respectively.

Strong acidities used to be considered as a prerequisite for the formation of highly ordered mesoporous silica when mineral acids were used as acid sources. ${ }^{9}$ A high concentration of hydronium ions in the strongly acidic solution can catalyze hydrolysis of siloxane..$^{10-13,28}$ Moreover, strong acidities can induce intensive protonation of the PEO corona of triblock copolymer micelle, ${ }^{13,37}$ which offers strong electrostatic and hydrogen bonding interactions with silica species, facilitating the assembly of silica precursors around the micelle template. ${ }^{38-43}$ Under weak acidities actually only a very small amount of silica precipitated if hydrochloric acid or nitric acid was used as the acid source due to limited silica condensation reactions. However, it has been shown in this work that some largemolecule polyhydroxy carboxylic acids can also lead to highly ordered mesostructure even under moderately weak acidic conditions $(\mathrm{pH} 1.5-2.0)$. We tentatively propose that the 


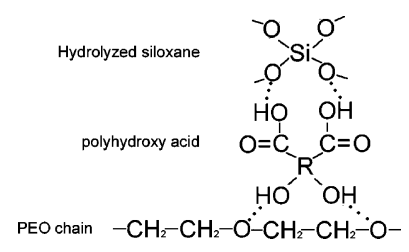

Figure 4. Example for polyhydroxy carboxylic acid molecules bridging silica species and template phases by hydrogen bond.
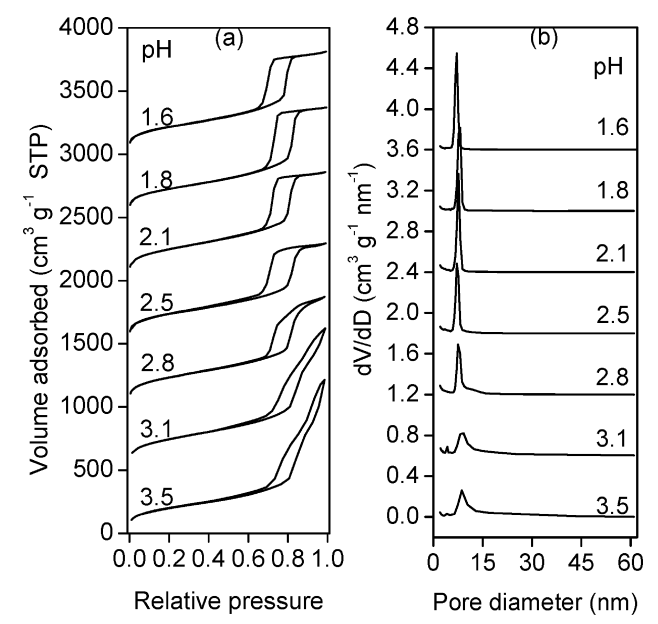

Figure 5. (a) Nitrogen sorption isotherms and (b) mesopore size distribution profiles of $\mathrm{MS}_{\mathrm{t}}$ silicas synthesized at various $\mathrm{pH}$ values adjusted by tartaric acid.

promoting effect of polyhydroxy carboxylic acids on the formation of ordered mesostructure arises primarily from their mediating effect on the interactions between the silica precursors and the triblock copolymers, as is exemplified in Figure 4. This assumption might be supported by the FT-IR spectra of the assynthesized materials before calcinations (Figure S1 in Supporting Information). The FT-IR spectra indicate that both assynthesized $\mathrm{MS}_{\mathrm{ci}}$ and $\mathrm{MS}_{\mathrm{t}}$ samples contain carboxyl groups, which could indicate that some organic acid molecules were incorporated into the silica-polymer hybrids. The molecules of both tartaric acid and citric acid bear several carboxyl and hydroxyl groups, and these polar groups can hydrogen-bond with both the copolymers and the silica species because their electropositive hydrogen atoms can have high affinity to the electronegative oxygen atoms in both the copolymer chains and the silica species. Therefore, the assembly forces between the silica species and the template phase can be strengthened due to the bridging capability of polyhydroxy carboxylic acid molecules, which can accelerate the accumulation of silica species around the charged PEO corona, kinetically prompt their condensation around the templates, and finally pack them into hexagonal structure. ${ }^{44}$ In addition, carboxylic acids are effective catalysts for the hydrolysis of siloxane,,${ }^{9,45}$ which is also quite favorable for the assembly reactions. But the small-molecule organic acids, such as acetic acid and oxalic acid, have much less polar groups in their molecules; thus their mediating effect on the assembly forces is too weak to promote the formation of ordered mesoporous materials.

3.2. Effect of Acidity and Acid Source on Textural Property. Figure 5 displays the nitrogen sorption isotherms and pore size distribution profiles of $\mathrm{MS}_{\mathrm{t}}$ silicas synthesized at various $\mathrm{pH}$ values. All the materials synthesized at $\mathrm{pH} \leq 2.5$ feature type IV isotherms with typical H1-type hysteresis loop, indicating their typical characteristic of mesoporous materials. ${ }^{46}$ The remarkably sharp capillary condensation steps of isotherms at relative pressure of $P / P_{0}=0.75-0.85$ and narrow sharp pore
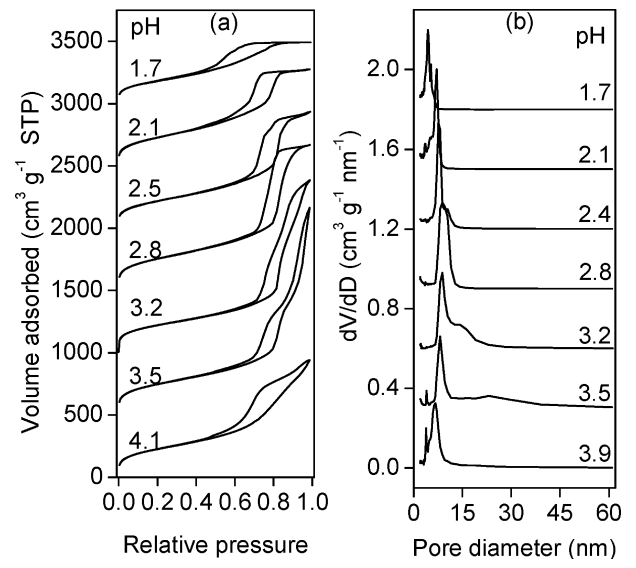

Figure 6. (a) Nitrogen sorption isotherms and (b) mesopore size distribution profiles of $\mathrm{MS}_{\mathrm{a}}$ silica synthesized at various $\mathrm{pH}$ values adjusted by acetic acid.

Table 1. Textural Properties of Various Mesoporous Silicas

\begin{tabular}{lcccc}
\hline sample & $A_{\mathrm{BET}}\left(\mathrm{m}^{2} / \mathrm{g}\right)$ & $V_{\text {total }}\left(\mathrm{cm}^{3} / \mathrm{g}\right)$ & $d_{\mathrm{BJH}}(\mathrm{nm})$ & $\mathrm{fwhm}^{a}(\mathrm{~nm})$ \\
\hline $\mathrm{MS}_{\mathrm{t}}-1.6$ & 756 & 1.3 & 7.3 & 1.1 \\
$\mathrm{MS}_{\mathrm{t}}-2.8$ & 835 & 1.3 & 7.5 & 1.4 \\
$\mathrm{MS}_{\mathrm{ci}}-1.8$ & 802 & 1.4 & 8.1 & 1.0 \\
$\mathrm{MS}_{\mathrm{ci}}-2.8$ & 864 & 1.3 & 7.5 & 1.4 \\
$\mathrm{MS}_{\mathrm{a}}-2.7$ & 784 & 1.8 & 8.8 & 3.5 \\
$\mathrm{MS}_{\mathrm{ox}}-2.8$ & 925 & 1.6 & 9.6 & 2.9
\end{tabular}

${ }^{a}$ Full width at half-maximum of the $\mathrm{BJH}$ peak.

size distribution peaks reveal that the materials have uniform cylindrical mesopore channels. Increase of the synthesis values from $\mathrm{pH} 2.8$ to 3.5 leads to the formation of H3-type hysteresis loop in the nitrogen isotherm, ${ }^{46}$ and the low and broad pore distribution peak of $\mathrm{MS}_{\mathrm{t}}-3.5$ indicates it features decreased mesopore uniformity. A similar evolution trend with acidity can be observed for $\mathrm{MS}_{\mathrm{ci}}$ silica (Figure S2 in Supporting Information).

Figure 6 shows the nitrogen sorption isotherms and pore size distribution profiles of $\mathrm{MS}_{\mathrm{a}}$ silicas. Typical H1-type hysteresis loop can be obtained only at $\mathrm{pH} \sim 2.7$ with the use of acetic acid. Furthermore, the mesopore uniformity of the materials synthesized using small-molecule organic acid seems inferior to that of those synthesized using large-molecule organic acids. It can clearly be seen from pore size distribution peaks and their full-width at half-maxium height (fwhm) listed in Table 1 that the former has broader peaks. Similar results can also be obtained with the use of oxalic acid (Figure S3 in Supporting Information).

The physical properties of some samples synthesized under organic acid are listed in Table 1. Apparently, the pore sizes and pore volumes of the materials synthesized with the use of citric acid or tartaric acid are relatively smaller than those synthesized with the use of acetic acid or oxalic acid, which might suggest that the micelle conformation of triblock copolymer is influenced by the acid molecules. It is well-established that the hydrophobic PPO core of the copolymer micelle contributes primarily to the formation of mesoporosity. ${ }^{1,2,42}$ And the hydrophilic PEO corona region contributes to the formation of part of the framework wall. ${ }^{42,47}$ However, some researchers demonstrated that a fraction of the PEO segments also contributed to the formation of the mesoporosity, ${ }^{47}$ some EO units adjacent to the PPO core were not solvated by water depending on the acidities, and they are incorporated into the hydrophobic part. As discussed previously, the polar hydroxyl groups and carboxyl groups of these large-molecule organic acids can 
(a)

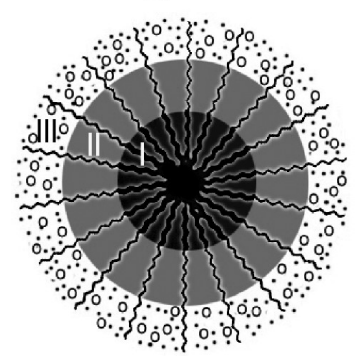

I: Hydrophobic PPO core (b)

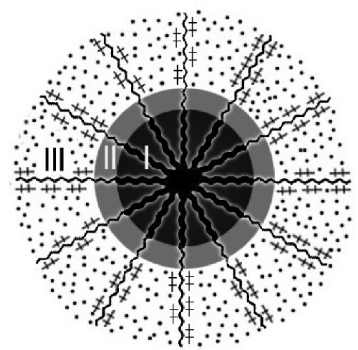

II: Non-solvated PEO shell III: Hydrated PEO corona
Figure 7. Illustration for the influence of the acid sources on the hydrophobicity of the copolymer micelle template: (a) in the presence of small-molecule carboxylic acids; (b) in the presence of polyhydroxy carboxylic acids. $\ddagger$, Molecules of polyhydroxy carboxylic acid; $\bigcirc$, molecules of small-molecule carboxylic acid; $\boldsymbol{\bullet}$, molecules of solvent water.

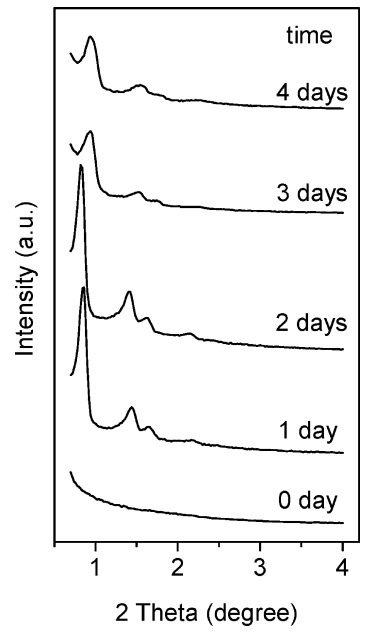

Figure 8. XRD patterns of $\mathrm{MS}_{\mathrm{t}}-1.6$ synthesized with low-temperature aging at $35{ }^{\circ} \mathrm{C}$ for different time.

interact strongly with the PEO chains by hydrogen bonding, which favors them to penetrate into the inner part of the PEO shell of the copolymer micelles, being accompanied with the intrusion of solvent water, ${ }^{48}$ thus producing a larger hydrated corona and a concurrently reduced hydrophobic volume, as illustrated in Figure 7. In contrast, the small-molecule carboxylic acids cause a lesser degree of hydration in the PEO shell and a larger hydrophobic volume; consequently the products have relatively larger pore diameters. The decrease of the hydrophobic volume of triblock copolymer micelle in the presence of polar molecules was indirectly revealed in previous work, ${ }^{49}$ where the researchers found that the addition of urea that borne polar amido groups into the aqueous solution of copolymer resulted in the decrease of the aggregation number of copolymer micelle. It could be expected that the copolymer molecules would become loosely aggregated in the micelles with the decrease of the aggregation number, ${ }^{27}$ which might also facilitate the intrusion of water into the inner part of the PEO block and concomitantly decrease the hydrophobic volume.

3.3. Effect of Low-Temperature Aging on Material Structure. In this work, the synthesis solutions were first aged at a low temperature of $35{ }^{\circ} \mathrm{C}$ prior to high-temperature hydrothermal treatment at $100{ }^{\circ} \mathrm{C}$. The influence of the lowtemperature aging on the structure of the final products was studied. Figure 8 presents the XRD patterns of the $\mathrm{MS}_{\mathrm{t}}$ silicas synthesized at $\mathrm{pH} 1.6$ with low-temperature aging at $35^{\circ} \mathrm{C}$ for different time. The material synthesized without low-temperature
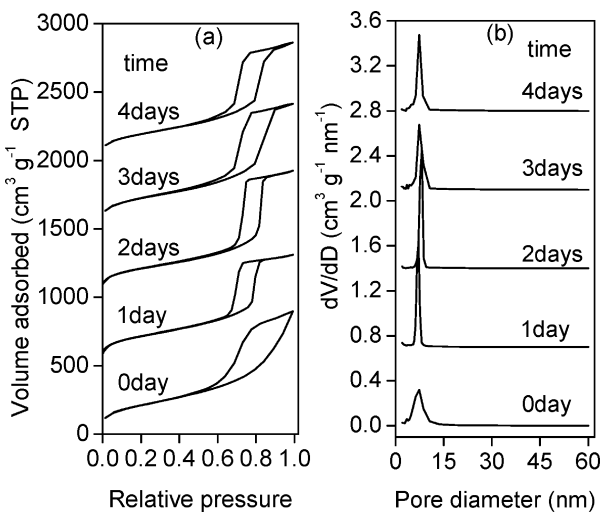

Figure 9. (a) Nitrogen sorption isotherms and (b) mesopore size distribution profiles of the $\mathrm{MS}_{\mathrm{t}}-1.6$ synthesized with low-temperature aging at $35^{\circ} \mathrm{C}$ for different time.

aging does not show any long-range order. In contrast, the material synthesized with a low-temperature aging for 2 days exhibits the most intensive diffraction peaks, indicating it has the best mesostructural ordering. But excess low-temperature aging time of more than 3 days seems to decrease the ordering as evidenced by the gradual reduction in diffraction intensity.

Figure 9 presents the nitrogen sorption isotherms and pore size distribution profiles of the $\mathrm{MS}_{\mathrm{t}}$ silica synthesized at $\mathrm{pH}$ 1.6 with low-temperature aging at $35^{\circ} \mathrm{C}$ for different time. The isotherm of product synthesized without aging at low temperature features a H2-type hysteresis loop, which is indicative of a broad pore size distribution. ${ }^{50,51}$ In contrast, the other materials synthesized with enough low-temperature aging time have uniform cylindrical mesopores as reflected by the H1-type hysteresis loop on their sorption isotherms and narrow pore size distributions. ${ }^{50,51}$

This indicates that the induction period at a relatively lower temperature $\left(35{ }^{\circ} \mathrm{C}\right)$ is necessary to form highly ordered mesostructure. If the initial limpid synthesis solutions were directly subjected to high-temperature aging at $100{ }^{\circ} \mathrm{C}$, the intensified thermal motions of both silica species and copolymer molecules would break seriously the weak hydrogen bonding as well as electrostatic assembly interactions, ${ }^{28,50,51}$ resulting in the formation of disordered mesoporous materials. On the other hand, it should be mentioned that well-ordered mesostructure was not always fully acquired during the induction period of the synthesis. ${ }^{52}$ The development of periodic mesostructure is fulfilled by continued reorganization of the aggregates in further hydrothermal aging. ${ }^{40-43,53,54}$ Because the condensation reaction is relatively slower at low temperature, ${ }^{52}$ the initial precipitates formed during the induction period are expected to be flexible complexes with low condensation degree $^{52,55}$ It is known that the high temperature could drive the surfactant aggregates to form more ordered superstructures. ${ }^{56,57}$ As a result, during the subsequent aging at elevated temperature of $100{ }^{\circ} \mathrm{C}$, the flexible silica solids are capable of undergoing continuous reorganization along with the phase transformation of the copolymer micelles, and further polymerize to concrete solids with hexagonal structure. ${ }^{55,58,59}$ In this work, 2 days of low-temperature aging is optimal for the formation of the best ordered structure, but more than 3 days of low-temperature aging time causes a negative effect on the quality of the product. Excess aging at $35{ }^{\circ} \mathrm{C}$ possibly results in a somewhat densified rigid structure due to excessive cross-linking, which inhibits the further structural transformation at the high-temperature aging period. 


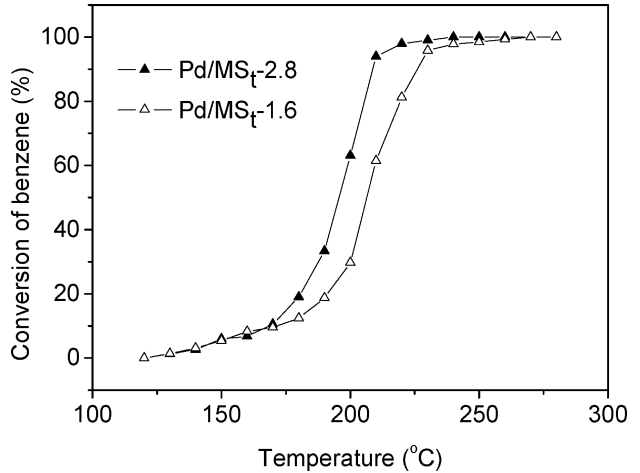

Figure 10. Ignition curves of benzene over palladium catalysts supported on mesoporous silicas.

\subsection{Effect of Material Morphology on Catalytic Activ-} ity. Because of the high surface areas and large pore sizes, SBA15 is expected to be a desirable catalyst support. In this work, the ordered silica with variable particle sizes controlled by the added amount of tartaric acid were used as support of palladium catalysts, and their catalytic performances were evaluated in the catalytic oxidation of benzene, one of the volatile organic pollutants of most concern from industrial emissions. The ignition curves of benzene over the catalysts are shown in Figure 10. The catalysts are capable of catalyzing the complete oxidation of benzene at a temperature of less than $250^{\circ} \mathrm{C}$, but it was ever revealed that the conversion of benzene was negligible even when the temperature was raised to $500{ }^{\circ} \mathrm{C}$ without catalysts. ${ }^{60}$ Furthermore, $\mathrm{MS}_{\mathrm{t}}-2.8$ composed of small particles seems superior to $\mathrm{MS}_{\mathrm{t}}-1.6$ with large particle size in the catalytic oxidation of benzene.

The catalytic removal of benzene is a complicated heterogeneous reaction, and comprehensive investigations are required to reveal the exact factors that govern the catalysis activity. Here we just tentatively explain the difference in the activity of the mesoporous catalysts in terms of the morphological properties of silica supports. For mesoporous molecular sieves based catalysts, their particle sizes might significantly affect the catalytic behaviors. ${ }^{34-36}$ For the preparation of supported catalyst by an impregnation method, a small particle size of the mesoporous silica support can afford good dispersion of active metals into the inner porosity, leading to high surface areas of active phases. ${ }^{61}$ Furthermore, a small particle favors the easy diffusion of reactants to the inner active sites. Thus, the palladium catalyst supported on $\mathrm{MS}_{\mathrm{t}}-2.8$ composed of the smallest particles exhibits better efficiency in the oxidation of benzene. However, studies on confinement effect are still required for clearly understanding the acting mechanism of the catalyst. ${ }^{62}$ It is very interesting that the morphology and pore structure can be tailored for specific applications by simply varying the synthesis acidities and acid sources.

\section{Conclusions}

Polyhydroxy carboxylic acids such as tartaric acid and citric acid have profound promoting effect on the synthesis of homogeneous ordered SBA- 15 under moderate acidities at $\mathrm{pH}$ $\leq 2.8$, but small-molecule organic acids such as acetic acid and oxalic acid do not. The ordered domains grow with increasing concentration of the polyhydroxy carboxylic acids. The several polar groups on the molecules of the polyhydroxy acids might promote the assembly forces by bridging the silica species and copolymer micelles via hydrogen bonding. The synthesis of SBA-15 with the use of polyhydroxy acids to adjust acidity can afford a safe, environment-friendly, and large-scale preparation method under the mild conditions. Furthermore, it is interesting that particle sizes of the mesoporous silicas can be easily tailored by adjusting the concentration of polyhydroxy carboxylic acids, which is desirable for catalysis or other applications due to the controllable mass transfer.

\section{Acknowledgment}

Financial support from the National Basic Research Program of China (Grant 2004CB719500), the National High Technology Research and Development Program of China (Grant 2006AA06A310), and the National Science Fund of China (Grants 20807050 and 20725723) is gratefully acknowledged. J.J.L. appreciates the scientific research start fund from the Chinese Academy of Sciences.

Supporting Information Available: Nitrogen sorption isotherms of $\mathrm{MS}_{\mathrm{ci}}$ and $\mathrm{MS}_{\mathrm{ox}}$ silica synthesized under various acidities. This material is available free of charge via the Internet at http://pubs.acs.org.

\section{Literature Cited}

(1) Zhao, D. Y.; Feng, J. L.; Huo, Q. S.; Melosh, N.; Fredrickson, G. H.; Chmelka, B. F.; Stucky, G. D. Triblock copolymer syntheses of mesoporous silica with periodic 50 to 300 angstrom pores. Science 1998, 279, 548.

(2) Zhao, D. Y.; Huo, Q. S.; Feng, J. L.; Chmelka, B. F.; Stucky, G. D. Nonionic triblock and star diblock copolymer and oligomeric surfactant syntheses of highly ordered hydrothermally stable mesoporous silica structures. J. Am. Chem. Soc. 1998, 120, 6024.

(3) Taguchi, A.; Schuth, F. Ordered mesoporous materials in catalysis. Microporous Mesoporous Mater. 2005, 77, 1 .

(4) Zhao, X. S.; Su, F. B.; Yan, Q. F.; Guo, W. P.; Bao, X. Y.; Lv, L.; Zhou, Z. C. Templating methods for preparation of porous structures. $J$. Mater. Chem. 2006, 16, 637.

(5) Soler-illia, G. J. de A. A.; Sanchez, C.; Lebeau, B.; Patarin, J. Chemical strategies to design textured materials: From microporous and mesoporous oxides to nanonetworks and hierarchical structures. Chem. Rev. 2002, 102, 4093.

(6) Davis, M. E. Ordered porous materials for emerging applications. Nature 2002, 417, 813.

(7) Schuth, F. Endo- and exotemplating to create high-surface-area inorganic materials. Angew. Chem., Int. Ed. 2003, 42, 3604.

(8) Hoffmann, F.; Cornelius, M.; Morell, J.; Froba, M. Silica-based mesoporous organic-inorganic hybrid materials. Angew. Chem., Int. Ed. 2006, $45,3216$.

(9) Hsu, Y. C.; Hsu, Y. T.; Hsu, H. Y.; Yang, C. M. Facile synthesis of mesoporous silica SBA-15 with additional intra-particle porosities. Chem. Mater. 2007, 19, 1120.

(10) Lin, H. P.; Kao, C. P.; Mou, C. Y.; Liu, S. B. Counterion effect in acid synthesis of mesoporous silica materials. J. Phys. Chem. B 2000, 104, 7885 .

(11) Bao, X. Y.; Zhao, X. S.; Li, X.; Chia, P. A.; Li, J. A novel route toward the synthesis of high-quality large-pore periodic mesoporous organosilicas. J. Phys. Chem. B 2004, 108, 4684.

(12) Abdullah, A. Z.; Abdullah, H.; Bhatia, S. Synthesis, characterization and material evaluation of mesoporous silicas templated by a polyglycols triblock copolymer for catalytic applications. Mater. Chem. Phys. 2007, 103, 375.

(13) Bagshaw, S. A. The effect of dilute electrolytes on the formation of non-ionically templated [Si]-MSU-X mesoporous silica molecular sieves. J. Mater. Chem. 2001, 11, 831.

(14) Vinu, A.; Sawant, D. P.; Ariga, K.; Hossain, K. Z.; Halligudi, S. B.; Hartmann, M.; Nomura, M. Direct synthesis of well-ordered and unusually reactive FeSBA-15 mesoporous molecular sieves. Chem. Mater. 2005, 17, 5339.

(15) Li, W. J.; Huang, S. J.; Liu, S. B.; Coppens, M. O. Influence of the Al source and synthesis of ordered Al-SBA-15 hexagonal particles with nanostairs and terraces. Langmuir 2005, 21, 2078.

(16) Li, Y.; Zhang, W. H.; Zhang, L.; Yang, Q. H.; Wei, Z. B.; Feng, Z. C.; Li, C. Direct synthesis of Al-SBA-15 mesoporous materials via hydrolysis-controlled approach. J. Phys. Chem. B 2004, 108, 9739. 
(17) Chen, S. Y.; Cheng, S. Acid-free synthesis of mesoporous silica using triblock copolymer as template with the aid of salt and alcohol. Chem. Mater. 2007, 19, 3041.

(18) Feng, P. Y.; Bu, X. H.; Pine, D. J. Control of pore sizes in mesoporous silica templated by liquid crystals in block copolymercosurfactant-water systems. Langmuir 2000, 16, 5304.

(19) Schmidt-Winkel, P.; Yang, P. D.; Margolese, D. I.; Chmelka, B. F.; Stucky, G. D. Fluoride-induced hierarchical ordering of mesoporous silica in aqueous acid-syntheses. Adv. Mater. 1999, 11, 303.

(20) Kim, J. M.; Han, Y. J.; Chmelka, B. F.; Stucky, G. D. One-step synthesis of ordered mesocomposites with non-ionic amphiphilic block copolymers: Implications of isoelectric point, hydrolysis rate and fluoride. Chem. Commun. (Cambridge) 2000, 2437.

(21) Cui, X. G.; Zin, W. C.; Cho, W. J.; Ha, C. S. Nonionic triblock copolymer synthesis of SBA-15 above the isoelectric point of silica $(\mathrm{pH}=2-$ 5). Mater. Lett. 2005, 59, 2257.

(22) Du, Y. C.; Lan, X. J.; Liu, S.; Ji, Y. Y.; Zhang, Y. L.; Zhang, W. P.; Xiao, F. S. The search of promoters for silica condensation and rational synthesis of hydrothermally stable and well ordered mesoporous silica materials with high degree of silica condensation at conventional temperature. Microporous Mesoporous Mater. 2008, 112, 225.

(23) Yu, C. Z.; Tian, B. Z.; Fan, B.; Stucky, G. D.; Zhao, D. Y. Salt effect in the synthesis of mesoporous silica templated by non-ionic block copolymers. Chem. Commun. (Cambridge) 2001, 2726.

(24) Qiao, S. Z.; Yu, C. Z.; Hu, Q. H.; Jin, Y. G.; Zhou, X. F.; Zhao, X. S.; Lu, G. Q. Control of ordered structure and morphology of largepore periodic mesoporous organosilicas by inorganic salt. Microporous Mesoporous Mater. 2006, 91, 59.

(25) Qiao, S. Z.; Yu, C. Z.; Xing, W.; Hu, Q. H.; Djojoputro, H.; Lu, G. Q. Synthesis and bio-adsorptive properties of large-pore periodic mesoporous organosilica rods. Chem. Mater. 2005, 17, 6172.

(26) Newalkar, B. L.; Komarneni, S. Control over microporosity of ordered microporous-mesoporous silica SBA-15 framework under microwavehydrothermal conditions: Effect of salt addition. Chem. Mater. 2001, 13, 4573.

(27) Kubo, S.; Kosuge, K. Salt-induced formation of uniform fiberlike SBA-15 mesoporous silica particles and application to toluene adsorption. Langmuir 2007, 23, 11761.

(28) Boissiere, C.; Larbot, A.; van der Lee, A.; Kooyman, P. J.; Prouzet, E. A new synthesis of mesoporous MSU-X silica controlled by a two-step pathway. Chem. Mater. 2000, 12, 2902.

(29) Jin, Z. W.; Wang, X. D.; Cui, X. G. Acidity-dependent mesostructure transformation of highly ordered mesoporous silica materials during a two-step synthesis. J. Non-Cryst. Solids 2007, 353, 2507.

(30) Jin, Z. W.; Wang, X. D.; Cui, X. G. Synthesis and morphological investigation of ordered SBA-15-type mesoporous silica with an amphiphilic triblock copolymer template under various conditions. Colloids Surf., A 2008, 316, 27.

(31) Luo, Y. M.; Hou, Z. Y.; Li, R. T.; Zheng, X. M. Rapid synthesis of ordered mesoporous silica with the aid of heteropoly acids. Microporous Mesoporous Mater. 2008, 109, 585.

(32) Colilla, M.; Balas, F.; Manzano, M.; Vallet-Regi, M. Novel method to enlarge the surface area of SBA-15. Chem. Mater. 2007, 19, 3099.

(33) Naskar, M. K.; Eswaramoorthy, M. Significant improvement in the pore properties of SBA-15 brought about by carboxylic acids and hydrothermal treatment. J. Chem. Sci. 2008, 120, 181.

(34) Ji, X. L.; Lee, K. T.; Monjauze, M.; Nazar, L. F. Strategic synthesis of SBA-15 nanorods. Chem. Commun. (Cambridge) 2008, 4288.

(35) Zhang, H.; Sun, J.; Ma, D.; Bao, X.; Klein-Hoffmann, A.; Weinberg, G.; Su, D.; Schlogl, R. Unusual mesoporous SBA-15 with parallel channels running along the short axis. J. Am. Chem. Soc. 2004, 126, 7440.

(36) Zhang, H.; Sun, J.; Ma, D.; Weinberg, G.; Su, D. S.; Bao, X. Engineered complex emulsion system: Toward modulating the pore length and morphological architecture of mesoporous silicas. J. Phys. Chem. B 2006, 110, 25908.

(37) Yang, B.; Guo, C.; Chen, S.; Ma, J. H.; Wang, J.; Liang, X. F.; Zheng, L.; Liu, H. Z. Effect of acid on the aggregation of poly(ethylene oxide)-poly(propylene oxide)-poly(ethylene oxide) block copolymers. $J$. Phys. Chem. B 2006, 110, 23068.

(38) Monnier, A.; Schuth, F.; Huo, Q.; Kumar, D.; Margolese, D.; Maxwell, R. S.; Stucky, G. D.; Krishnamurty, M.; Petroff, P.; Firouzi, A.; Janicke, M.; Chmelka, B. F. Cooperative formation of inorganic-organic interfaces in the synthesis of silicate mesostructures. Science 1993, 261 , 1299.

(39) Cheng, C. F.; Luan, Z. H.; Klinowski, J. The role of surfactant micelles in the synthesis of the mesoporous molecular sieve MCM-41. Langmuir 1995, 11, 2815 .
(40) Regev, O. Nucleation events during the synthesis of mesoporous materials using liquid crystalline templating. Langmuir 1996, 12, 4940.

(41) Flodstrom, K.; Wennerstrom, H.; Alfredsson, V. Mechanism of mesoporous silica formation. A time-resolved NMR and TEM study of silica-block copolymer aggregation. Langmuir 2004, 20, 680.

(42) Imperor-Clerc, M.; Davidson, P.; Davidson, A. Existence of a microporous corona around the mesopores of silica-based SBA-15 materials templated by triblock copolymers. J. Am. Chem. Soc. 2000, 122, 11925.

(43) Zholobenko, V. L.; Khodakov, A. Y.; Impéror-Clerc, M.; Durand, D.; Grillo, I. Initial stages of SBA-15 synthesis: An overview. Adv. Colloids Interfac. 2008, 142, 67.

(44) Ruthstein, S.; Schmidt, J.; Kesselman, E.; Talmon, Y.; Goldfarb, D. Resolving intermediate solution structures during the formation of mesoporous SBA-15. J. Am. Chem. Soc. 2006, 128, 3366.

(45) Green, W. H.; Le, K. P.; Grey, J.; Au, T. F.; Sailor, M. J. White phosphors from a silicate-carboxylate sol-gel precursor that lack metal activator ions. Science 1997, 276, 1826.

(46) Sing, K. S. W.; Evrett, D. H.; Haul, R. A. W.; Moscou, L.; Pierotti, R. A.; Rouqerol, J.; Siemieniewska, T. Reporting physisorption data for gas/solid systems with special reference to the determination of surface area and porosity. Pure Appl. Chem. 1985, 57, 603.

(47) Smarsly, B.; Polarz, S.; Antonietti, M. Preparation of porous silica materials via sol-gel nanocasting of nonionic surfactants: A mechanistic study on the self-aggregation of amphiphiles for the precise prediction of the mesopore size. J. Phys. Chem. B 2001, 105, 10473.

(48) Baute, D.; Goldfarb, D. Interaction of nitrates with pluronic micelles and their role in the phase formation of mesoporous materials. J. Phys. Chem. C 2007, 111, 10931.

(49) Desai, P. R.; Jain, N. J.; Sharma, R. K.; Bahadur, P. Effect of additives on the micellization of PEO/PPO/PEO block copolymer F127 in aqueous solution. Colloids Surf., A 2001, 178, 57.

(50) Tanev, P. T.; Pinnavaia, T. J. A neutral templating route to mesoporous molecular-sieves. Science 1995, 267, 5199.

(51) Tanev, P. T.; Pinnavaia, T. J. Mesoporous silica molecular sieves prepared by ionic and neutral surfactant templating: A comparison of physical properties. Chem. Mater. 1996, 8, 2068.

(52) Huo, Q. S.; Margolese, D. I.; Ciesla, U.; Feng, P. Y.; Gier, T. E.; Sieger, P.; Leon, R.; Petroff, P. M.; Schuth, F.; Stucky, G. D. Generalized synthesis of periodic surfactant-inorganic composite materials. Nature 1994, 368,317

(53) Chen, S.-Y.; Tang, C.-Y.; Chuang, W.-T.; Lee, J.-J.; Tsai, Y.-L.; Chan, J. C. C.; Lin, C.-Y.; Liu, Y.-C.; Cheng, S. A facile route to synthesizing functionalized mesoporous SBA-15 materials with platelet morphology and short mesochannels. Chem. Mater. 2008, 20, 3906.

(54) Linton, P.; Alfredsson, V. Growth and morphology of mesoporous SBA-15 particles. Chem. Mater. 2008, 20, 2878.

(55) Kosuge, K.; Sato, T.; Kikukawa, N.; Takemori, M. Morphological control of rod-and fiberlike SBA-15 type mesoporous silica using watersoluble sodium silicate. Chem. Mater. 2004, 16, 899.

(56) Mortensen, K.; Pedersen, J. S. Structural study on the micelle formation of poly(ethylene oxide)-poly(propylene oxide)-poly(ethylene oxide) triblock copolymer in aqueous solution. Macromolecules 1993, 26, 805.

(57) Alexandridis, P.; Holzwarth, J. F.; Hatton, T. A. Micellization of poly(ethylene oxide)-poly(propylene oxide)-poly(ethylene oxide) triblock copolymers in aqueous solutions: Thermodynamics of copolymer association. Macromolecules 1994, 27, 2414.

(58) Boissiere, C.; Larbot, A.; Bourgaux, C.; Prouzet, E.; Bunton, C. A. A study of the assembly mechanism of the mesoporous MSU-X silica twostep synthesis. Chem. Mater. 2001, 13, 3580.

(59) Li, J. J.; Xu, X. Y.; Hao, Z. P.; Zhao, W. Mesoporous silica supported cobalt oxide catalysts for catalytic removal of benzene. J. Porous Mater. 2008, 15, 163.

(60) Li, J. J.; Jiang, Z.; Hao, Z. P.; Xu, X. Y.; Zhuang, Y. H. Pillared laponite clays-supported palladium catalysts for the complete oxidation of benzene. J. Mol. Catal. A 2005, 225, 173.

(61) Li, J. J.; Xu, X. Y.; Jiang, Z.; Hao, Z. P.; Hu, C. Nanoporous silicasupported nanometric palladium: Synthesis, characterization, and catalytic deep oxidation of benzene. Environ. Sci. Technol. 2005, 39, 1319.

(62) Goettmann, F.; Sanchez, C. How does confinement affect the catalytic activity of mesoporous materials. J. Mater. Chem. 2007, 17, 24.

Received for review January 20, 2009 Revised manuscript received May 21, 2009 Accepted May 24, 2009

IE900100G 